\title{
Measurement of Serum Prolactin Level in Vitiligo Patients and its Correlation with Prolactin Gene Polymorphism
}

E.M.Sanad $^{1}$, S.H.Ahmed ${ }^{1}$, Y.M. Marei ${ }^{2}$ and A.K.Ahmad ${ }^{1}$

${ }^{1}$ Dermatology, Andrology and Venerology Dept., Faculty of Medicine, Benha Univ., Benha, Egypt

${ }^{2}$ Medical Biochemistry Dept., Faculty of Medicine, Benha Univ., Benha, Egypt

E-Mail:asmaa@gmail.com

\begin{abstract}
Vitiligo is a procured depigmentation issue including around $1-2 \%$ of populace overall ignoring age, race and sexual orientation. Prolactin is a chemical discharged by lactotrop cells in pituitary organ. PRL appears to have extra capacities in skin science and hair follicle development also. The point of this examination was to assess serum levels of Prolactin (PRL) in patients with vitiligo in contrast with sound control and to survey its relationship with prolactin quality polymorphism and vitiligo seriousness. This forthcoming case-control study was led on forty patients grumbling of vitiligo, and forty age and sex coordinated sound volunteers. Patients were enlisted from outpatient facility of Dermatology, Venereology and Andrology Department of Benha University Hospitals.There was non-huge contrast among patients and control bunches with respect to age, BMI, SBP, DBP, sex or uncommon propensities. There was non-huge distinction as respect serum PRL in patients bunch contrasted with control gathering. In patients gathering, PRL quality polymorphism demonstrated noncritical connection with sex, exceptional propensities and family ancestry. In patients gathering, PRL quality polymorphism indicated non-critical relationship with age, SBP, DBP, serum PRL, term of vitiligo and VASI score. While it demonstrated a huge connection with BMI. In patients gathering, serum PRL indicated non-huge relationship with age, BMI, SBP, DBP, term of vitiligo and VASI score.
\end{abstract}

Keywords: Prolactin, Vitiligo, Gene, Polymorphism.

\section{Introduction}

Vitiligo is an obtained depigmentation issue including around $1-2 \%$ of populace overall ignoring age, race and sexual orientation. The victims are described by particular melanocyte annihilation in the skin. Diminished number of melanocytes in the skin results in depigmented patches in various pieces of body, arise generally in second to fourth many years of life [1].

Precise pathogenesis of the illness is as yet not selfevident, hereditary qualities and anxious deformities, neuropeptides, biochemical and auto-damaging instruments just as viral disease and hormonal or cytokine cooperations are accused to be the triggers [2].

Prolactin (PRL) is a chemical discharged by lactotrop cells in pituitary organ. PRL appears to have extra capacities in skin science and hair follicle development also. This notable chemical has been acknowledged in a few articles as an immunostimulatory factor in immune system issues like psoriasis and lupus erythematosus [3].

Prolactin builds the blend of interferon gamma (IFN$\gamma$ ) and interleukin 2 (IL-2) by T-aide 1 lymphocytes (Th1). Besides, PRL enacts T-partner 2 lymphocytes (Th2) with autoantibody creation. Numerous investigations assessed the clinical importance of PRL in various skin sicknesses with clashing outcomes. The human prolactin quality is arranged on the short arm of chromosome 6 , near the significant histocompatibility complex (MHC) [4].

Past investigations have discovered linkage disequilibrium between MHC qualities known to be related with rheumatoid joint pain and foundational lupus erythematosus (SLE), raising the likelihood that polymorphisms in the upstream administrative locales of the human prolactin quality, which maybe decide levels of lymphocyte-explicit record, may be related with sickness. The marvel of utilitarian advertiser locale polymorphisms has been seen in other cytokine qualities.
In the IL-10 quality, for instance, the connections of 3 diverse single-nucleotide polymorphisms (SNPs) inside the advertiser district have been analyzed. For this situation, exceptionally transcriptionally dynamic haplotypes have been characterized and have been discovered to be related with SLE [5].

There are not really modest bunch of investigates zeroing in on the part of prolactin in the pathophysiology of vitiligo and its relationship with prolactin quality polymorphism.

The point of the current examination was to assess serum levels of prolactin in patients with vitiligo in contrast with solid control and to evaluate its connection with prolactin quality polymorphism and vitiligo seriousness.

\section{Patients and methods}

This case control study was conducted on eighty patients (forty patients complaining of vitiligo, and forty age and sex matched healthy volunteers). Patients were recruited from outpatient clinic of Dermatology, Venereology and Andrology Department of Benha University Hospitals during the period from October 2019 to May 2020.

\section{Inclusion criteria}

All patients between 18 and 60 years of age with stable vitiligo vulgaris will be enrolled in this study.

\section{Exclusion criteria}

Any patient with any of the following condition was excluded from this study:

- Patients with any chronic hepatic disorder or immune deficiency

- Kidney disorders

- Autoimmune disorders as SLE and thyroiditis.

- Thyroid gland diseases as Hashimotos thyroiditis 
- Current pregnancy or breast feeding which already changes ordinary serum hormone concentrations

- Using oral contraceptives, dopamine receptors blockers, atypical antipsychotics, metoclopramide, methyldopa, histamine antagonists, imipramine, selective serotonin reuptake inhibitors (SSRIs), calcium channel blockers and addiction.

This study was approved by the Research Ethical Committee of Benha Faculty of Medicine. Before taking blood samples, a written informed consent was taken from each patient and normal volunteer in the control group.

All participants were divided into two groups:

- Group A: forty patients with vitiligo.

- Group B: forty age and sex matched volunteers as controls group.

All patients were subjected to full history taking, complete clinical examination, and laboratory investigations for detection of serum level of prolactin and prolactin gene polymorphism.

Statistical analysis

Information were genuinely depicted as far as mean \pm standard deviation (Mean \pm SD), middle and reach, or frequencies (number of cases) and rates when suitable.
Examination of mathematical factors between the investigation bunches was finished utilizing Student $t$ test (t-test) for free examples in contrasting 2 gatherings when typically dispersed. The test insights used to gauge the factual relationship, or relationship, between two persistent factors is Pearson's connection coefficient (rtest). The non-parametric test (Spearman's Rho-test) was utilized to quantify the strength of relationship between two factors. Fisher's careful test (F-test) was utilized to know whether the extents for one factor are distinctive among estimations of the other variable. Precise test was utilized rather when the normal recurrence is under 5. Pvalues under 0.05 was considered genuinely huge. All measurable figurings were organized and investigated utilizing PC programs SPSS (Statistical Package for the Social Science; SPSS Inc., Chicago, IL, USA) adaptation 16 for Microsoft Windows.

\section{Results}

The present study was conducted on 40 patients suffering from vitiligo and 40 age and sex matched healthy volunteers. Age, sex, and BMI showed no significant difference between both studied groups $(\mathrm{p}>$ $0.05)$ as shown in Table (1).

Table (1) Comparison between patients and control groups regarding age, BMI and sex.

\begin{tabular}{lccccc}
\hline & & Patients & Control & T-test t value & P value \\
\hline Age & Range & $20-52$ & $20-52$ & 0.418 & 0.677 \\
BMI & Mean \pm SD & $34.4 \pm 8.1$ & $33.7 \pm 7.4$ & & 0.799 \\
& Range & $19-26$ & $19-25$ & -0.281 & \\
& Mean \pm SD & $22.6 \pm 1.9$ & $22.7 \pm 2$ & & P value \\
\hline Sex & & Patients & Control & Chi-square $X^{2}$ value & 0.000 \\
& Male & $20(50 \%)$ & $20(50 \%)$ & & 1.000 \\
\hline
\end{tabular}

Serum prolactin was measured in both studied groups. In patients' group, prolactin level ranged from 0.1399 to 2.7482 $\mathrm{ng} / \mathrm{ml}($ Mean $\pm \mathrm{SD}=0.598 \pm 0.454 \mathrm{ng} / \mathrm{ml})$ while in control group it ranged from 0.0488 to $2.5384 \mathrm{ng} / \mathrm{ml}($ Mean $\pm \mathrm{SD}=0.756 \pm 0.579 \mathrm{ng} / \mathrm{ml})$. There was insignificant difference regarding serum prolactin in patients group compared to control group $(\mathrm{P}=0.178)$ as shown in Fig $(1)$.

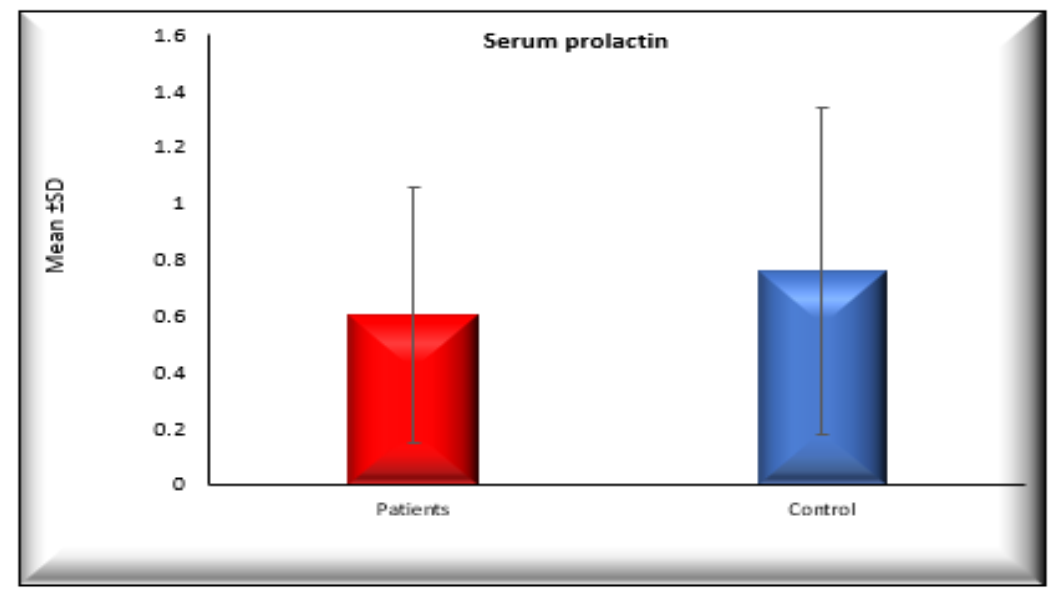

Fig. (1) Comparison between patients and control groups as regard serum prolactin.

Receiver operating characteristic (ROC) curve analysis of serum prolactin was conducted for discrimination between vitiligo and control groups. Poor AUC was found $(\mathrm{AUC}=0.440)$. At best cut of value of maximized sensitivity and specificity $(=0.486 \mathrm{ng} / \mathrm{mL})$, sensitivity was $50 \%$, specificity was $45 \%$ as shown in Fig (2). 


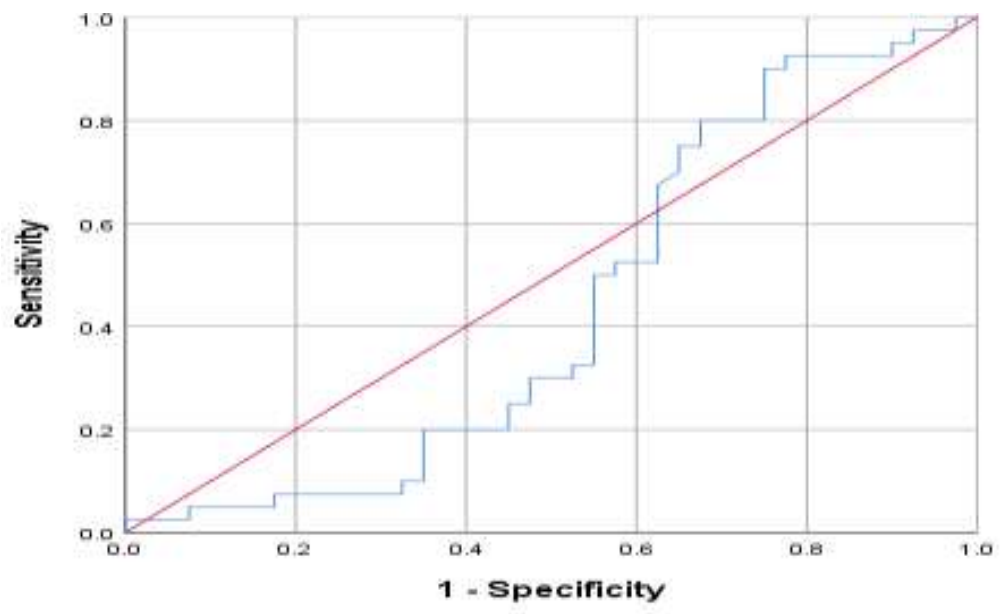

Fig. (2) ROC curve of prolactin for discrimination between vitiligo and control groups.

The genotype in patients' group showed that $3(7.5 \%)$ had GG genotype, 37 (92.5\%) had GT genotype. While in control group $8(20 \%)$ had GG genotype, $32(80 \%)$ had GT. The most common genotype found in both groups was GT. There were no statistically significant differences between the two groups $(\mathrm{P}=0.105)$ as shown in Table $(2)$.

Table (2) Comparison between patients and controls regarding prolactin gene polymorphism.

\begin{tabular}{llllll}
\hline $\begin{array}{lllll}\text { PRL gene } \\
\text { polymorphism }\end{array}$ & Patients & Control & Total & $\begin{array}{l}\text { Chi-square } \\
X^{2} \text { value }\end{array}$ & P value \\
\cline { 2 - 4 } $\mathbf{N}(\boldsymbol{\%})$ & $\mathbf{N}(\boldsymbol{\%})$ & $\mathbf{N}(\%)$ & & \\
G/T & $3(3.75 \%)$ & $8(10 \%)$ & $11(13.75 \%)$ & & \\
Total & $37(46.25 \%)$ & $32(40 \%)$ & $69(86.23 \%)$ & & 0.105 \\
\hline
\end{tabular}

Logistic regression analysis of the two genotypes of the prolactin gene revealed no significant difference between the two genotypes in both groups as shown in Table (3).

Table (3) Prolactin G/T genotypes and alleles in the studied groups.

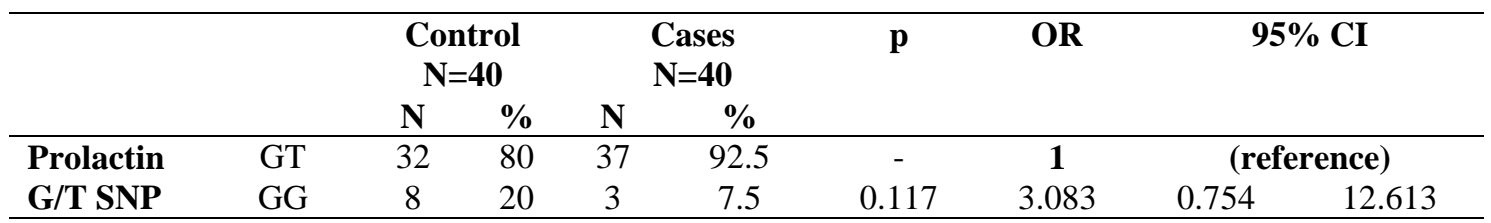

$\mathrm{OR}$, odds ratio; CI, confidence interval; logistic regression test was used.

\section{Discussion}

In this planned case-control study, all vitiligo patients were chosen from outpatient center of Dermatology, Venereology and Andrology division of Benha University Hospitals.

In the current examination, BMI demonstrated no critical contrast between both considered gatherings. Additionally to our outcome, Procaccini et al. [6] discovered that vitiligo didn't give off an impression of being related with a high $\mathrm{BMI}$; weight probably won't be a danger factor for vitiligo, as opposed to most immune system sicknesses which are fundamentally connected with corpulence. Likewise, Karadag et al. [7] detailed no critical contrast in BMI between vitiligo gathering and control gathering. The creators proposed that in spite of the fact that insulin opposition is expanded in patients with vitiligo, various instruments other than stoutness might be answerable for insulin obstruction in such patients. Besides, Dragoni et al. [8] indicated that vitiligo didn't seem, by all accounts, to be related with a high BMI. This absence of an affiliation could be because of a high articulation of p53 and a high centralization of changing development factor- $\beta$ (TGF- $\beta$ ) in vitiligo patients.

Despite the fact that, stoutness is a proinflammatory condition wherein hypertrophic adipocytes and fat tissueinhabitant cells add to increment circling levels of proinflammatory cytokines [9]. There have been contradictiry results about the relationship of BMI and vitiligo.

In the current investigation, PRL level demonstrated non-critical distinction in patients, bunch contrasted with control gathering.

Like the current examination, Bozkurt et al.[10] recorded that PRL levels had no genuinely huge distinction between the two gatherings. Despite the fact 
that the frequency of different autoantibodies and immune system illnesses in patients determined to have hyperprolactinemia was found to build, there were no measurably critical consequences of PRL in the etiopathogenesis of vitiligo.

As opposed to the current examination, Elsherif et al. [3] showed that the serum PRL level was fundamentally higher in patients than controls. De Bellis et al. [11] showed that PRL has various immunostimulatory impacts and advances autoimmunity. PRL builds the combination of IL-6 and IL-2 which are proinflammatory cytokines that assume a significant job in melanocytic cytotoxicity in vitiligo patients. Numerous specialists detailed high IL-6 levels in vitiligo patients. IL-6 was created by mononuclear cells, which could prompt the outflow of ICAM-1 (intercellular cell bond atoms) on melanocytes, which may then encourage leukocytemelanocyte associations, prompting polyclonal B-cell actuation along these lines expanding autoantibody creation, prompting immunological harm of melanocytes. Additionally, PRL enacts Th2 lymphocytes with autoantibody creation and upgrades immunoglobulin creation which may add to expanded autoreactivity [11].

In the current investigation, we found that serum PRL demonstrated non-critical connection with sex, exceptional propensities and family ancestry in the patient, gathering.

Likewise, Gönül et al.[12] didn't locate a critical distinction as far as sex and family ancestry with PRL levels between vitiligo patients and control gathering.

Also Elsherif et al. [3] uncovered no critical distinction in patient's serum PRL and their sex.

Additionally, Bozkurt et al. [10] found that there was no measurably critical contrast in sex despite the fact that there were higher qualities in female patients, particularly those with summed up sort of vitiligo.

In the current work, serum PRL indicated non-critical connection with age, BMI, SBP, DBP, span of vitiligo and VASI score in patient gathering.

In concurrence with the current examination, Elsherif et al. [3] uncovered no huge contrast in patients' serum PRL level and their age and illness spans.

Likewise Gönül et al. [12] didn't locate a critical distinction in term old enough and length of ailment with PRL levels between vitiligo patients and control gathering.

In the current examination, the ROC bend investigation of serum PRL was led for separation among vitiligo and control gatherings. Helpless AUC was discovered (AUC $=0.440)$. Best case scenario, cut of estimation of amplified affectability and explicitness $(=0.486 \mathrm{ng} / \mathrm{mL})$, affectability was half, particularity was $45 \%$.

As far as we could possibly know, there is no distributed examination with respect to the quality polymorphism of PRL in vitiligo sickness.

The current information demonstrated the genotype of PRL in patients bunch was $3(7.5 \%)$ of patients had GG genotype, 37 (92.5\%) of patients had GT genotype.
While in benchmark group $8(20 \%)$ had GG genotype, $32(80 \%)$ had GT. The most genotype found in the two patients and control bunches was GT. There were no genuinely huge contrasts between the two gatherings.

Prolactin polymorphism has been concentrated in a few immune system sicknesses, for example, different sclerosis, psoriatic joint pain, foundational sclerosis (SSc), rheumatoid joint inflammation and SLE [13].

Our outcomes came in concurrence with Montoyadiaz, et al. [13] in Czech and Central Mexico populaces with SLE, where they didn't discover a relationship between the PRL - 1149 G/T SNP and SLE.

\section{Conclusion}

Since PRL is additionally created locally in the skin, it is assumed that neighborhood arrival of chemical can't hit the limit of serum distinguished degrees of PRL bringing about typical fixations in vitiligo. As, There wasn,t any critical connection between's serum convergence of PRL and vitiligo event or its seriousness so PRL has no job in pathogenesis of vitiligo.

\section{References}

[1] S. E. Radvar, Z. Tehranchi, M. Partovikia, A. Kazeminejad, and S. H. S. Amini, "Assessment of prolactin levels in vitiligo patients and healthy controls and it's association with severity of disease," Pigm Disord, Vol.2(155), PP. 427-2376, 2014.

[2] A. Taylor, M. Pawaskar, S. L. Taylor, R. Balkrishnan, S. R. Feldman, "Prevalence of pigmentary disorders and their impact on quality of life: a prospective cohort study," J. Cosmet. Dermatol, Vol.7(3), PP. 164-168, 2008.

[3] N. A. Elsherif, A. I. El-Sherif, and S. A. El-Dibany. Serum prolactin levels in dermatological diseases: a case-control study," J. Dermatology Dermatologic Surg., Vol.19(2), PP. 104-107, 2015.

[4] K. Foitzik, K. Krause, F.Conrad, M. Nakamura, W. Funk, R. Paus."Human scalp hair follicles are both a target and a source of prolactin, which serves as an autocrine and/or paracrine promoter of apoptosis-driven hair follicle regression," Am. J. Pathol, Vol.168(3), PP. 748-756, 2006.

[5] A. Stevens et al. "Characterization of a prolactin gene polymorphism and its associations with systemic lupus erythematosus," Arthritis Rheum.Vol.44(10), PP. 2358-2366, 2001.

[6] C. Procaccini et al. "Obesity and susceptibility to autoimmune diseases,” Expert Rev. Clin. Immunol, Vol.7(3), PP. 287-294, 2011.

[7] A. S. Karadag, E. Tutal, and D. T. ERTUgRUL. Insulin resistance is increased in patients with vitiligo," Acta Derm. Venereol., Vol.91(5), PP. 541-544, 2011.

[8] F. Dragoni . No Association between Vitiligo and Obesity: A Case-Control Study," Med. Princ. Pract, Vol.26(5), PP. 421-426, 2017.

[9] K. Makki, P. Froguel, and I. Wolowczuk. Adipose tissue in obesity-related inflammation and insulin 
resistance: cells, cytokines, and chemokines," Int. Sch. Res. Not, Vol.2013 (3), PP. 321-501, 2013.

[10] E.Ç. Bozkurt, H. Bozkurt, A. Yılmaz, and D. Atacan. Serum-prolactin levels in patients with vitiligo, Vol.2(10), PP. 432-470, 2016.

[11] A.De Bellis, A.Bizzarro, R.Pivonello, G.Lombardi, A.Bellastella. "Prolactin and autoimmunity," Pituitary.Vol.8(1), PP. 25-30, 2005.
[12] M.Gönül, U.Gül, C.Kılınç, S. K. Cakmak, "Prolactine levels in the patients with vitiligo," J. Eur. Acad. Dermatology Venereol, Vol.23(12), PP. 1454-1455, 2009.

[13] E. Montoya-Díaz . Prolactin Promoter Polymorphism (- $1149 \mathrm{G} / \mathrm{T})$ is Associated with ANTI-DNA Antibodies in Mexican Patients with Systemic Lupus Erythematosus," Immunol. Invest, Vol.40(6), PP. 614-626, 2011. 